\title{
In Situ Visual Observation of Fracture Processes in Several High-Performance Fibers
}

\author{
Matthew Hudspeth • Ben Claus • Niranjan Parab • \\ Boonhim Lim • Weinong Chen · Tao Sun • \\ Kamel Fezza
}

Received: 5 November 2014/Accepted: 8 February 2015/Published online: 18 February 2015

(C) Society for Experimental Mechanics, Inc 2015

\begin{abstract}
Three different high-performance fibers have been imaged in situ during Kolsky bar tensile loading using two different techniques, namely optical microscopy and phase contrast imaging (PCI). Kevlar ${ }^{\circledR} \mathrm{KM} 2$, Dyneema ${ }^{\circledR}$ SK76, and S-2 Glass ${ }^{\circledR}$ fibers have been pulled using an instrumented Kolsky bar, thereby shedding light on the failure process of each fiber type. Both the $\operatorname{Kevlar}^{\circledR} \mathrm{KM} 2$ fiber and Dyneema ${ }^{\circledR}$ SK76 fiber exhibit rupture defined by varying degrees of fibrillation, with the former typically showing longer fibrillated ends than the latter. S-2 Glass ${ }^{\circledR}$ failure was found to exhibit a brittle fracture mode at a single point, although post-mortem analysis commonly yielded disintegration of the fiber gauge length, which is concluded to occur post the initial break due to fiber snap back or bending. Finally the efficacy of utilizing the PCI technique to achieve higher levels of spatial and temporal resolution is discussed.
\end{abstract}

Keywords Aramid fiber $\cdot$ Yarn $\cdot$ Fabrics/textiles $\cdot$ Impact behavior

\section{Introduction}

High-performance fibers possess profound mechanical stiffness and strength values in their longitudinal direction and are therefore used in a vast number of engineering

M. Hudspeth · B. Claus · N. Parab · B. Lim · W. Chen $(\square)$ School of Aeronautics and Astronautics, Purdue University, West Lafayette, IN 47907, USA

e-mail: wchen@purdue.edu

T. Sun $\cdot$ K. Fezza

Advanced Photon Source, Argonne National Laboratory, Argonne, IL 60439, USA applications. Coupled with their low density $(\sim 1-2.4 \mathrm{~g} /$ $\mathrm{cm}^{3}$ ), they are most routinely employed in products requiring high strength and high stiffness, namely composite structures such as helicopter blades and sporting equipment, as well as in woven form such as anti-spall linings, mooring lines, and ballistic vests. Therefore, large amounts of testing have been performed on the longitudinal tensile properties of said fibers, which is generally defined by a linear-elastic stress-strain curve [1-5]. This is due to the extremely high level of orientation present for polymeric fibers, resulting in crystallinity values of $75-95 \%[2,3]$. Upon loading, these fiber types exhibit a linear-elastic stress-strain response, typically followed by a brittle fracture being defined by fibrillation for polymer fibers [6] and a point break for glass fibers [7]. Fibrillation is thought to occur due to the low transverse bonding of the molecular chains or fibrils, which is defined by Van der Waals attraction and/or hydrogen bonding [8]. It is thus highly unlikely that the ultimate strength of polymer fibers has been achieved, as failure most likely occurs due to interchain slippage, rather than chain scission [9-12]. Indeed, current achievable strengths seen in production of ultra high molecular weight polyethylene (UHMWPE) fibers are 3-4 GPa [13], which is only one-sixth to one-tenth of the theoretical fiber strength [3]. In order to determine the theoretical limit of an entire fiber rather than just a single molecular chain or fibril, it is necessary to ascertain an understanding of the fiber microstructure, as well as an understanding of the failure process, which can verify model predictions as well as determine if the model exhibits the proper failure mode. Therefore, an understanding of the failure phenomenon becomes of great importance, yet herein lies the main difficulty for failure analysis of high-performance fibers; rupture analysis is solely performed via post-mortem static imaging, as their size and 
time period of failure limit high-speed imaging visualization.

The essence of the post-mortem approach is to look at both pre- and post-failed fiber fracture images via optical microscopy or SEM, and then to combine this information with a load-deformation response in efforts to conjecture a proposed failure phenomenon [5, 14]. While this is a reasonable approach for the trained eye, it still leaves room for error, especially when rupture morphologies become complex or if the material does not exhibit a typical bulk response seen in common material testing. For example, it has been previously alluded to that aramid fibers exhibit a possible necking behavior during failure due to post-mortem rupture morphologies showing a very fine point break [2], which as will be described, is just an alternate form of the common failure mode, namely fibrillation. Furthermore, this method also becomes inadequate when examining high-strength glass fibers, which upon tensile failure, shatter into many pieces, thereby leaving the true fracture surface unrecoverable post deformation [7, 15].

To the authors' knowledge, no work in the literature has yet shown the failure process of high-performance fiber during the actual loading procedure. Lack of this in situ analysis is inherently due to the fine nature of these highperformance fibers, which are typically only $10-30 \mu \mathrm{m}$ in diameter. Furthermore, the failure process of ballistic fibers is dynamic in nature, thereby rendering imaging of the failure sequence during quasi-static testing quite difficult as the number of tests required to capture the said event would be extremely time prohibitive. Thus, it is the goal of this work to show the possibility of imaging high-performance fiber in situ, during a dynamic loading process. Two techniques will be discussed, namely optical light microscopy and phase contrast imaging (PCI). The capability of both of these techniques will be shown via Kolsky bar tensile testing of Kevlar ${ }^{\circledR}$ KM2, Dyneema ${ }^{\circledR}$ SK76, and S-2 Glass ${ }^{\circledR}$ followed by a brief analysis of the failure process from each fiber type. Finally, improvements in temporal and spatial resolution of the two imaging techniques will also be discussed.

\section{Experimental}

\section{Materials and Loading Method}

Single fiber fracture studies have been performed on three different fiber types: Kevlar ${ }^{\circledR}$ KM2, Dyneema ${ }^{\circledR}$ SK76, and $\mathrm{S}$-2-glass ${ }^{\circledR}$. Fibers were extracted from the as-received yarns and then attached to aluminum mounting fixtures. Said fixtures were designed to minimize sample gauge length, aiding in the likelihood of catching the dynamic fracture event within the active imaging window. Typical gauge lengths were roughly $200-400 \mu \mathrm{m}$ subject to the tolerance of the sample holder. Fiber ends were carefully fixed to the metal substrates using an appropriate epoxy, with extreme efforts placed on the minimization of epoxy wicking into the sample gauge length.

The mechanism used to deliver a dynamic loading to the sample was a miniature Kolsky bar apparatus, which can be seen in Fig. 1. As opposed to a traditional Kolsky bar, the miniature device ignores the use of a transmission bar, as the majority of the incident waveform is reflected back down the incident bar due to the large impedance mismatch between the fiber and loading bar end, rendering the traditional method of measuring the transmitted signal from strain gauges on the transmission bar impossible. Thus, for typical single fiber longitudinal tension testing, the transmission bar is replaced with a dynamic force transducer, thereby allowing for accurate determination of the sample loading history. In the case of this experimental approach, which is designed to capture the failure process of a single high-performance fiber, the load cell was not always used to detect the dynamic force history, due to space constraints of the optical assemblage, which will be explained below.

Upon adequate curing of the adhesive, the sample assemblage was loaded into the miniature Kolsky bar apparatus and prior to testing, a load isolating arm was removed from the metal sample holder in order to expose the fiber specimen to free loading between the incident bar and the rigid force transducer/backstop. Using a gas firing system, the incident bar was loaded with a concentric brass striker, thereby giving the desired loading pulse with proper pulse shaping. A typical loading waveform can be seen in Fig. 2. Collection of the loading pulse was performed with a data acquisition unit capable of outputting a 1.5 V TTL signal, which was then used to trigger the highspeed imaging device in both of the visualization techniques described below.

\section{Optical Light Method}

Fibers have been pulled in longitudinal tension on a miniature Kolsky bar outfitted with a visual light optical imaging capability as seen in Fig. 1a. Due to the extremely fine nature of these ballistic fibers $(\sim 10-30 \mu \mathrm{m})$, highspeed imaging with a traditional camera lens system was impossible. Thus, the aforementioned Kolsky bar apparatus has been outfitted with a light-microscope in order to magnify the fiber diameter to a level that is possible to collect on a CCD recording device. The total magnification of the microscope system was $20 \times$, with the microscope lens and camera lens adaptor possessing magnifications of $2 \times$ and $10 \times$, respectively. In order to focus the camera system onto the fiber sample, the entire Kolsky bar 

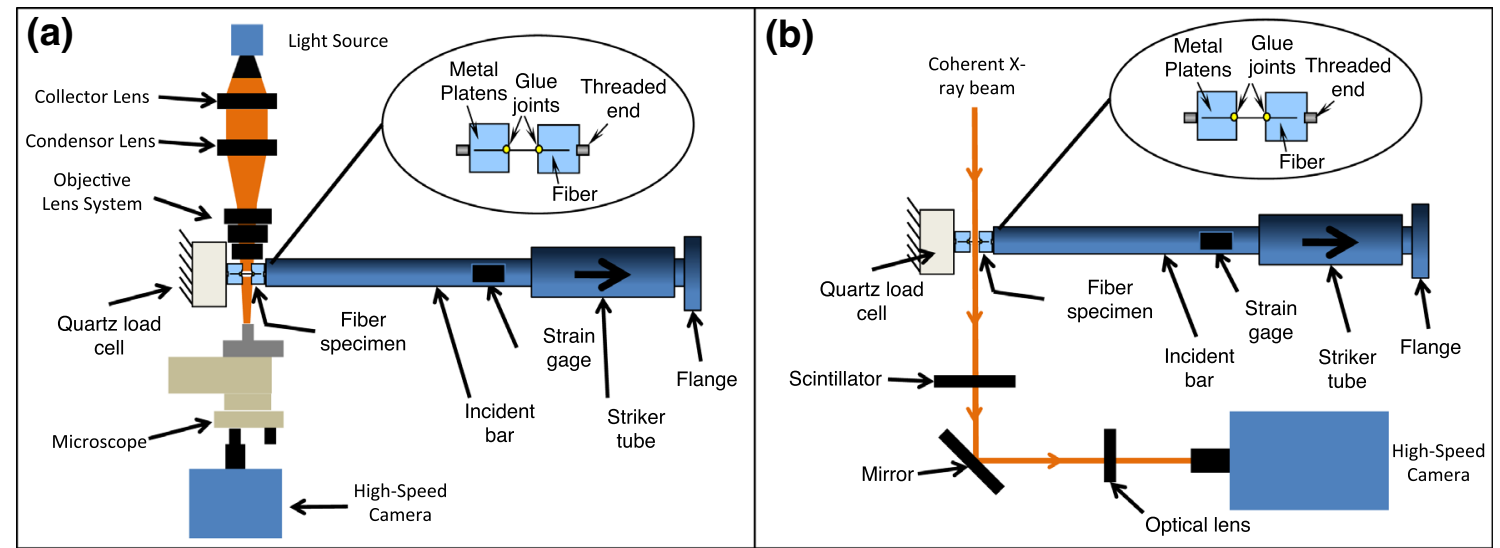

Fig. 1 Schematic of the miniature tension Kolsky bar coupled with the high rate imaging apparatus: a optical light microscopy and b PCI



Fig. 2 Oscilloscope record of the incident waveform (red) and the resulting TTL output (blue) from the oscilloscope used to trigger the high-speed imaging system (Color figure online)

apparatus was outfitted with a 3-axis stage, thereby allowing movement of the fiber sample with respect to the stationary imaging system (very much similar to the traditional lab optical microscopes). A high-speed Cordin 550 camera capable of taking 32 1-MP images was used to capture the dynamic failure event. This camera system possesses 32 stationary CCDs that are illuminated by a rotating platter possessing mirrors that direct the incoming light sequentially onto the various CCDs, thereby allowing full-frame resolution regardless of recording frame rate and magnification. Finally, in order to gain enough light to capture the dynamic failure event onto the CCDs of the high-speed camera, a high photon-flux flash bulb was used to illuminate the fiber sample. A light focusing lens system was employed to direct the flash down onto the fiber sample via a five lens system. A 2.5 in convex lens possessing a focal length of 2 in was first used to collect the light coming from the flash source. This collected light was then condensed down with a similar lens onto an objective triple lens system utilizing convex lenses of 1 in diameter and having focal lengths of 1.5, 0.75, and 0.75 in., ordered from upstream to downstream, respectively. Greatest success of this objective lens system was found when almost all flash light was directed into the end of the imaging microscope lens. As previously described, the incident waveform used to load the fiber sample was detected with strain gauges on the incident bar, and then captured with an oscilloscope data recorder. A $1.5 \mathrm{~V}$ TTL signal with a $50 \mathrm{~ns}$ delay from the oscilloscope trigger was then output to the camera, and by utilizing the camera software, an appropriate time delay (due to the travel time of the waveform down the incident bar) was used to capture the dynamic failure event at frame rates ranging between 200,000 and 300,000 fps (frames per second).

It is important to note that the fineness of these fibers inherently limits the resolution of light microscopy, as the wavelength of visual light is one-two and zero orders of magnitude less than the fiber diameter and possible fractured artifacts' minor thicknesses, respectively. Thus utilization of this first visual technique must be implemented with caution, as ruptured surface artifacts of the fracturing process may not be resolvable.

\section{Phase Contrast Imaging}

Due to the deficiency in spatial resolution of the optical light microscopy method, PCI has been employed to capture the dynamic failure process of single fibers using a synchrotron X-ray source, as X-rays inherently possess wavelengths several orders of magnitude less than visible light. It has been previously shown that PCI can be used to track internal/external failure processes of materials subjected to high loading rates using a Kolsky bar apparatus 
[16] or gas gun system [17-21]. In the present work, a Kolsky bar apparatus has been integrated with the X-ray source present in beamline 32-ID at APS, which utilizes an undulator A that possesses a period and length of $2.4 \mathrm{~m}$ and $3.3 \mathrm{~cm}$, respectively. The Kolsky bar was placed $40 \mathrm{~m}$ downstream of the X-ray source perpendicular to the beam path, and the X-ray beam size was controlled with adjustable slits, thereby allowing for beam size control in both the $\mathrm{x}$ - and $\mathrm{y}$-directions. In this set of experiments, the slit opening size was $1 \times 0.7 \mathrm{~mm}$ in the $\mathrm{x}$ - and $\mathrm{y}$-directions, respectively. By altering the gap of the undulator (30-40 $\mathrm{mm}$ for these tests), the spectrum characteristics of the X-ray source were varied, thereby providing an increase or decrease in photon flux, thus ensuring negligible damage to the fiber mechanical properties. Verification of negligible beam damage was verified by subsequent quasistatic tension experiments following ASTM C 1577-03 [22] on single fibers that were exposed to radiation doses similar to those of given in the PCI experiments, yielding unchanged filament elastic modulus, failure strain, and failure stress.

Due to the longevity of the loading process $(\sim 100 \mu \mathrm{s})$, a preliminary temporal resolution of $0.1-1 \mu \mathrm{s}$ is acceptable, which defines the necessary framing rate, and ultimately the X-ray integration time for a single frame. Thus, the frame rate is limited by the pulse electron bunch separation and electron travel time around the storage ring, which inherently selects the operation mode of the APS storage ring used during the experiments. It is important to note that these experiments were performed in tandem with other material class testing, thus the run mode selected was the APS hybrid mode, which inherently limits the maximum inter-frame separation to $3.68 \mu$ s when using the entire superbunch for camera exposure [17]. Once X-rays were produced by the undulator, they travelled down the beamline into the hutch in which the experiment was performed. As shown in Fig. 1b, the sample of interest was placed into the beamline path, and during the time of loading, X-rays were allowed to impinge upon the sample. Once they passed through the specimen, they were converted into light via a single crystal Lu3A15O12:Ce scintillator, which was placed $18 \mathrm{~cm}$ downstream of the sample. As the decay time of the scintillator was 45-55 ns, which was much less than the frame separation used in these experiments (minimum of $3.68 \mu \mathrm{s}$ ), the ghosting effect due to previous exposure was deemed negligible [17]. The light produced by the scintillator was then reflected by a $45^{\circ}$ mirror and then magnified by a $10 \times$ lens, ultimately being recorded by a high-speed camera. The camera used for the experiments was a Photron Fastcam SA1.1 12-bit CMOS camera, capable of achieving frame rates ranging from 5400 to $675,000 \mathrm{fps}$, with an inherent loss in image window size at elevated frame rates due to data transfer limitations. The camera was phase locked to an integer of the storage ring master clock, thereby providing recording windows during the X-ray passage. The camera was triggered using an external TTL (transistor-transistor logic) signal, being provided by a high-speed oscilloscope, which itself was triggered from strain gauges attached on the surface of the Kolsky bar. Further explanation of this procedure and the timing sequence is described in Hudspeth et al. [16] and a schematic of the experimental apparatus can be seen in Fig. 1 b.

Like the optical microscopy method, the Kolsky bar was placed on a position adjustable stand, thus allowing for proper location of the sample within the beamline path. Alignment of the sample with the Kolsky bar was performed with a 4-axis stage providing $\mathrm{x}-, \mathrm{y}$-, $\mathrm{z}$-displacement and rotation about the $\mathrm{z}$-axis. Sample assemblies previously described were used to ensure minimal gauge length of the fiber specimens, thereby allowing for visualization of the entire gage length within the camera view window.

\section{Results and Discussion}

High-performance fibers were pulled in tension and the fracture process was imaged using both optical light microscopy and PCI via the Kolsky bar apparatus shown in Fig. 1a, b, respectively. Resulting loading sequences from Kevlar $^{\circledR}$ KM2, Dyneema ${ }^{\circledR}$ SK76, and S-2 Glass ${ }^{\circledR}$ can be seen in Figs. 3 and 4, using the optical and PCI methods, respectively. Figure 3 a shows the loading history of a single Kevlar ${ }^{\circledR}$ KM2 fiber undergoing a fibrillation failure process. As can be seen from the panel sequence, fibrillation is an evolutionary process instilled with large amounts of longitudinal splitting of the fiber. This is believed to occur due to the weak transverse bonding present between the molecular chains and microfibrils, being defined by Van der Waals attraction and hydrogen bonding [8]. In contrast to this highdegree of fibrillation, Fig. 4a presents the failure process of a Kevlar ${ }^{\circledR}$ KM2 fiber when imaged with the PCI technique. During this loading process, the Kevlar ${ }^{\circledR}$ KM2 fiber presents a form of fibrillation, wherein both fracture surfaces break in a brittle fashion and along the axial direction, but only along one or two fracture planes, thereby resulting in a fine point break rupture morphology [4, 6]. This rupture morphology has previously led to the assumption that this fiber type may undergo a necking phenomenon [2], and as will be described and further demonstrated shortly, may be an invalid conclusion. Figure $3 \mathrm{~b}$ depicts the failure process presented by the Dyneema ${ }^{\circledR}$ SK76 fiber via imaging with optical microscopy. A low degree of fibrillation can be seen to occur during this fiber breakage, which is the typical rupture morphology presented by this fiber type. In contrast, Fig. 4b shows the failure process of two Dyneema ${ }^{\circledR}$ SK76 fibers, 


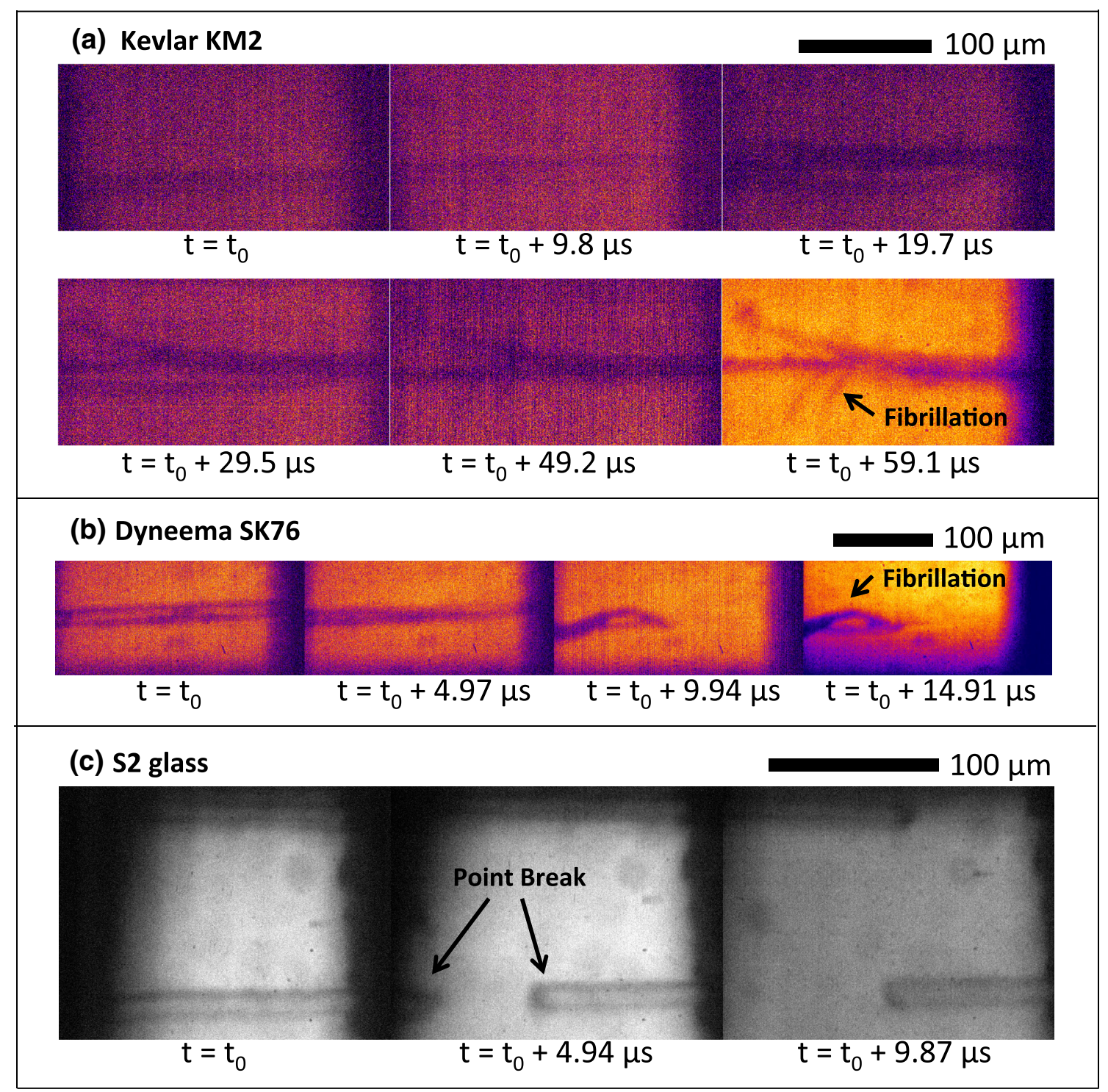

Fig. 3 High-rate optical microscopy images taken during the dynamic loading procedure, wherein: a $\operatorname{Kevlar}^{\circledR} \mathrm{KM} 2$ fiber is undergoing the fibrillation phenomenon. b Dyneema ${ }^{\circledR}$ SK76 is

with the top filament undergoing a mass amount of fibrillation during failure. Again, as with the Kevlar ${ }^{\circledR} \mathrm{KM} 2$ fiber, Dyneema ${ }^{\circledR}$ SK76 possesses very weak transverse bonding being defined by Van der Waals attraction between the long flexible PE molecular chains. Finally, both Figs. $3 \mathrm{c}$ and $4 \mathrm{c}$ show the failure process of S-2 Glass ${ }^{\circledR}$, which upon inspection, demonstrates that the fiber undergoes a brittle fracture process that is located at a single point along the fiber gauge length. It has been previously thought that high-performance glass fibers undergo a simultaneous disintegration process, wherein numerous cracks propagate through the filament during failure $[7,15]$. These images clearly show that this failure process is not occurring for S-2 Glass ${ }^{\circledR}$, rather the fiber is breaking in a single point, and further breakage noted undergoing a less pronounced degree of fibrillation. c Two S-2 Glass ${ }^{\circledR}$ fibers both fracture in a point break manner

in the aforementioned studies is most likely occurring during the unloading or bending of the fiber specimen post initial rupture. As the typical mode of fiber fracture analysis is performed via post-mortem static imaging, common fracture surfaces presented by each fiber type are shown in Fig. 5 . Fracture surface imaging from both the Kevlar ${ }^{\circledR} \mathrm{KM} 2$ fiber and the Dyneema ${ }^{\circledR}$ SK76 fiber were performed with SEM, while the rupture morphologies presented by the $\mathrm{S}-2$ Glass ${ }^{\circledR}$ fiber were imaged with optical microscopy. Both low- and high-rate fracture surfaces have been imaged so as to determine if there exists any rate effects in the failure process of these three different fiber types. With regards to the Kevlar ${ }^{\circledR}$ KM2 fiber, which can be seen in the top micrographs of Fig. 5, this fiber type exhibits rupture morphologies of either 


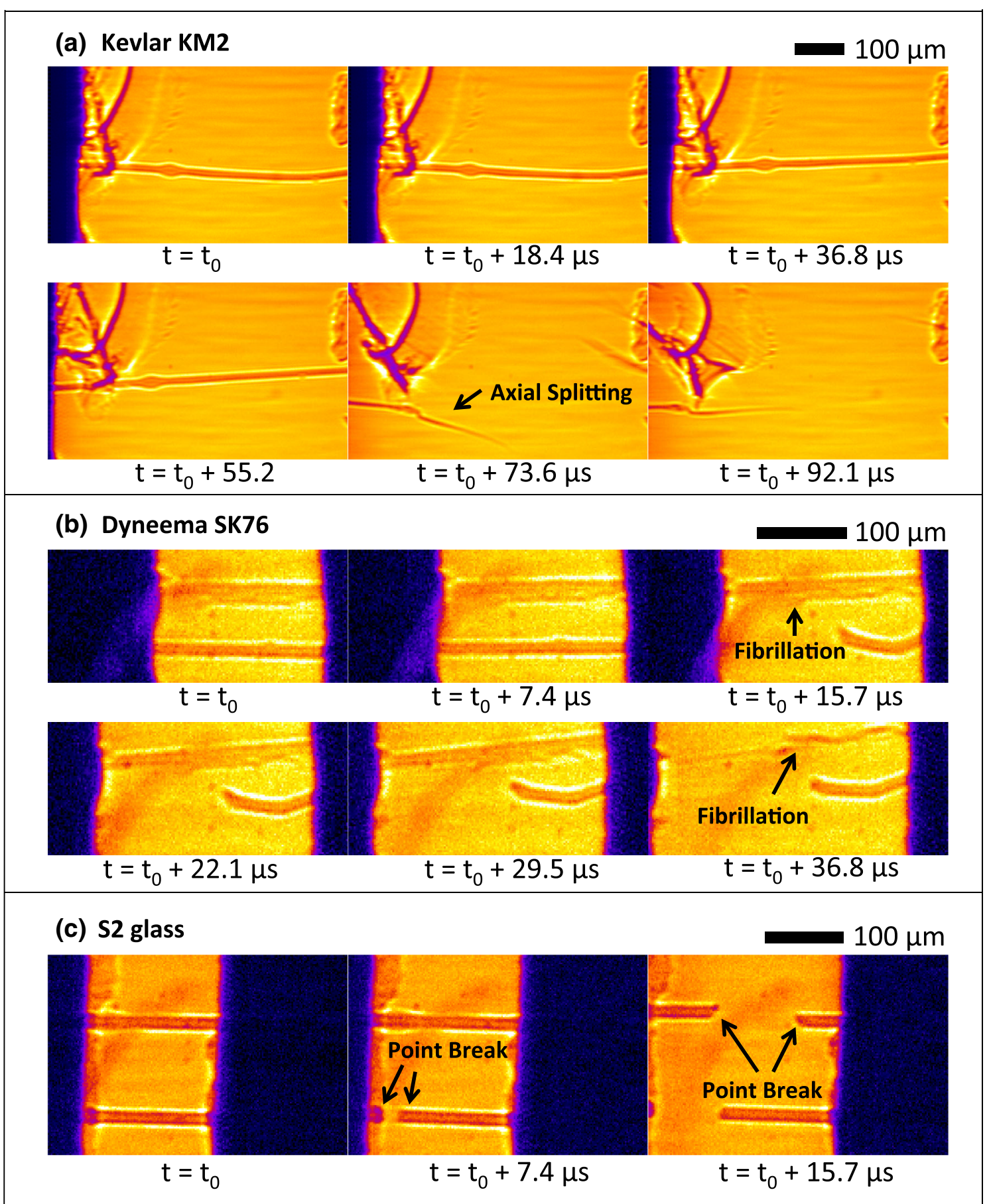

Fig. 4 PCI images taken during the dynamic loading procedure, wherein: a Kevlar ${ }^{\circledR}$ KM2 fiber is undergoing axial splitting. b The top Dyneema ${ }^{\circledR}$ SK76 is undergoing massive fibrillation while the bottom

fibrillation or axial splitting at both low and high strain-rates. As aforementioned, previous work has described the latter rupture morphology as a sort of necking behavior, as the failure surface does look to pull down to a fine breaking point as would occur during a ductile fracture process. Even though this sort of failure surface at first glance does
Sk76 fiber exhibits a less pronounced degree of fibrillation. c Two S-2 Glass ${ }^{\circledR}$ fibers both fracture in a point break manner

represent a plausible necking deformation mode, it is important to remember that the Kevlar $^{\circledR}$ KM2 fiber is quite brittle, possesses a failure strain of 3-4\%, and the majority of the molecular chains are oriented in the axial direction due to the manufacturing process. Indeed, as can be seen in the set of micrographs presented in Fig. 6, this failure process is 


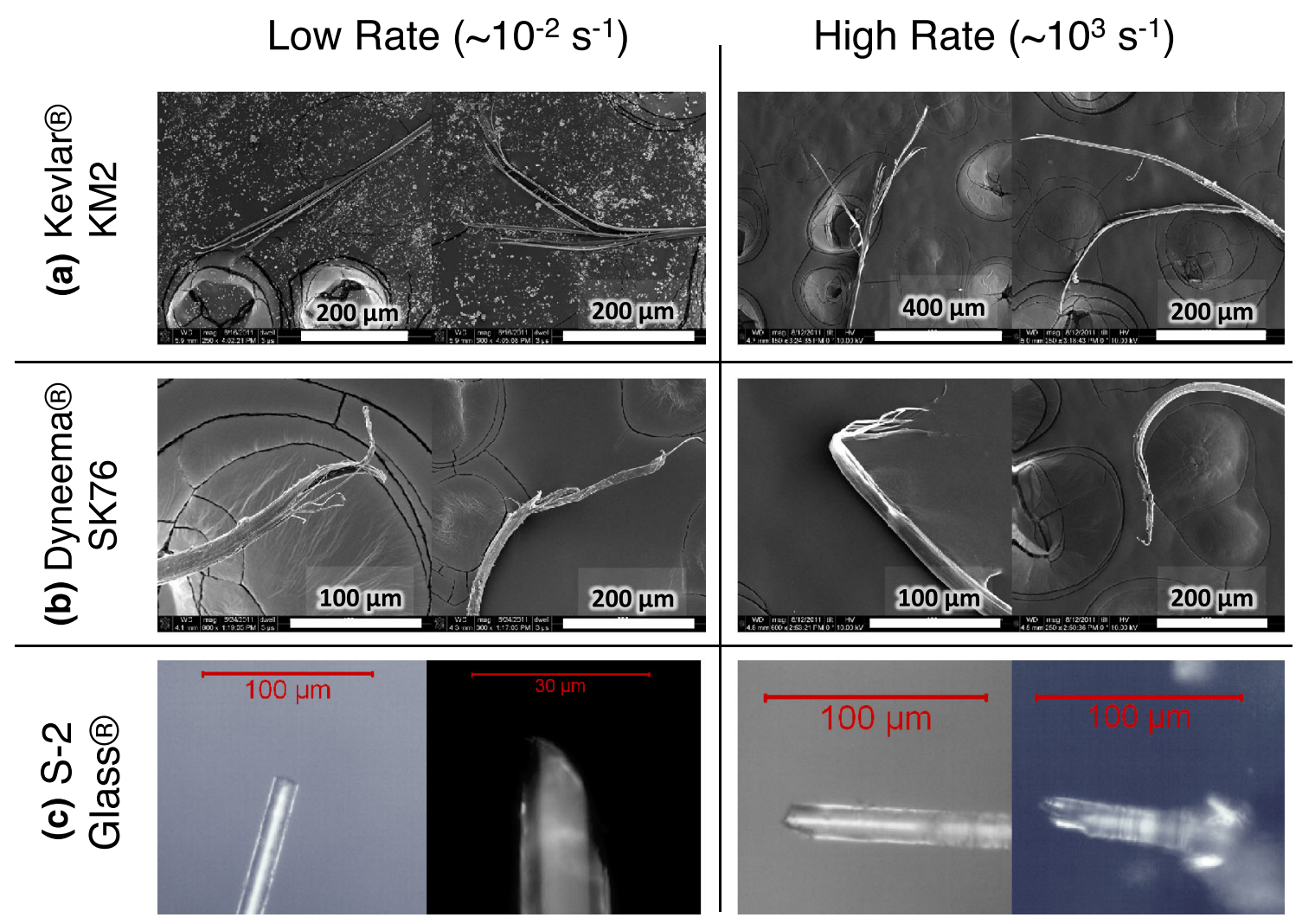

Fig. 5 Post-mortem micrographs of both low- and high-rate loading fracture morphologies: a SEM imaging of $\operatorname{Kevlar}^{\circledR} \mathrm{KM} 2$ exhibiting both fibrillation and axial splitting at low- and high-strain rates. b SEM imaging of Dyneema ${ }^{\circledR}$ SK76 fibers exhibiting various levels of fibrillation at both tested strain-rates. Very often the fibrillation process depicted ribbon-like structures, more than likely originating

certainly just a form of fibrillation, wherein only a single fibrillated end remains post failure. For a reference, Fig. 6 presents a case of the $\mathrm{Kevlar}^{\circledR} \mathrm{KM} 2$ fiber undergoing axial splitting, which was probed with both in situ imaging and post-mortem imaging. This failure process is an example of the axial splitting phenomenon that can be deduced from the post-mortem micrograph, which is seen in the bottom right inset of the image sequence. Herein the Kevlar ${ }^{\circledR} \mathrm{KM} 2$ fiber is undergoing a clear axial split ending in a very fine amount of fibrillation, rather than the occasionally assumed ductile fracture.

In the second set of post-mortem micrographs presented in Fig. 5, the typical rupture morphologies presented by the Dyneema ${ }^{\circledR}$ SK76 fiber can are shown. At both low and high strain-rate tensile failures, it is demonstrated that the fiber exhibits a fibrillation failure process, though not as drastic as that presented by the Kevlar ${ }^{\circledR}$ KM2 fiber. Rather than fine fibrillation, the Dyneema ${ }^{\circledR}$ SK76 fiber fracture surface looks to typically possess plate-like failure surfaces along with fine fibrillation [6,14]. Though not conclusive, in all micrographs found in this set of experiments, the plate-like features from the skin-core structure of the fiber. c Optical imaging the S-2 Glass ${ }^{\circledR}$ fiber from portions of fiber remaining post loading. Note, these fracture surfaces are more than likely not the proper initial failure surfaces, as this fiber type generally fractures into many fragments due to snap back or bending post initial fracture

originated from the skin portion of the fiber, which is a reasonable failure process, as it is well known that high-performance polymeric fibers typically possess a skin-core architecture [23-26]. It is important to note that mass fine fibrillation was also uncovered in a few of the post-mortem rupture morphologies, which can be seen in the first high-rate micrograph shown in the Dyneema ${ }^{\circledR}$ SK76 section of Fig. 5. This mass fibrillation process was also seen using the PCI technique, which has been previously described and is exhibited by the top fiber in Fig. 4b.

Finally, in the third set of post-mortem micrographs presented in Fig. 5, rupture morphologies of S-2 Glass ${ }^{\circledR}$ are presented. It is important to note that these failure surfaces were imaged from the slight portion of the specimen which remained on each side of the sample grid, as the majority of the fiber specimen within the gauge length was unrecoverable, which is commonly the case for high-performance glass fibers [7, 15]. Although the failure surfaces of these fiber ends clearly show a brittle fracture process, solely using postmortem failure surfaces to analyze the true fracture history of this fiber type becomes impossible as the 


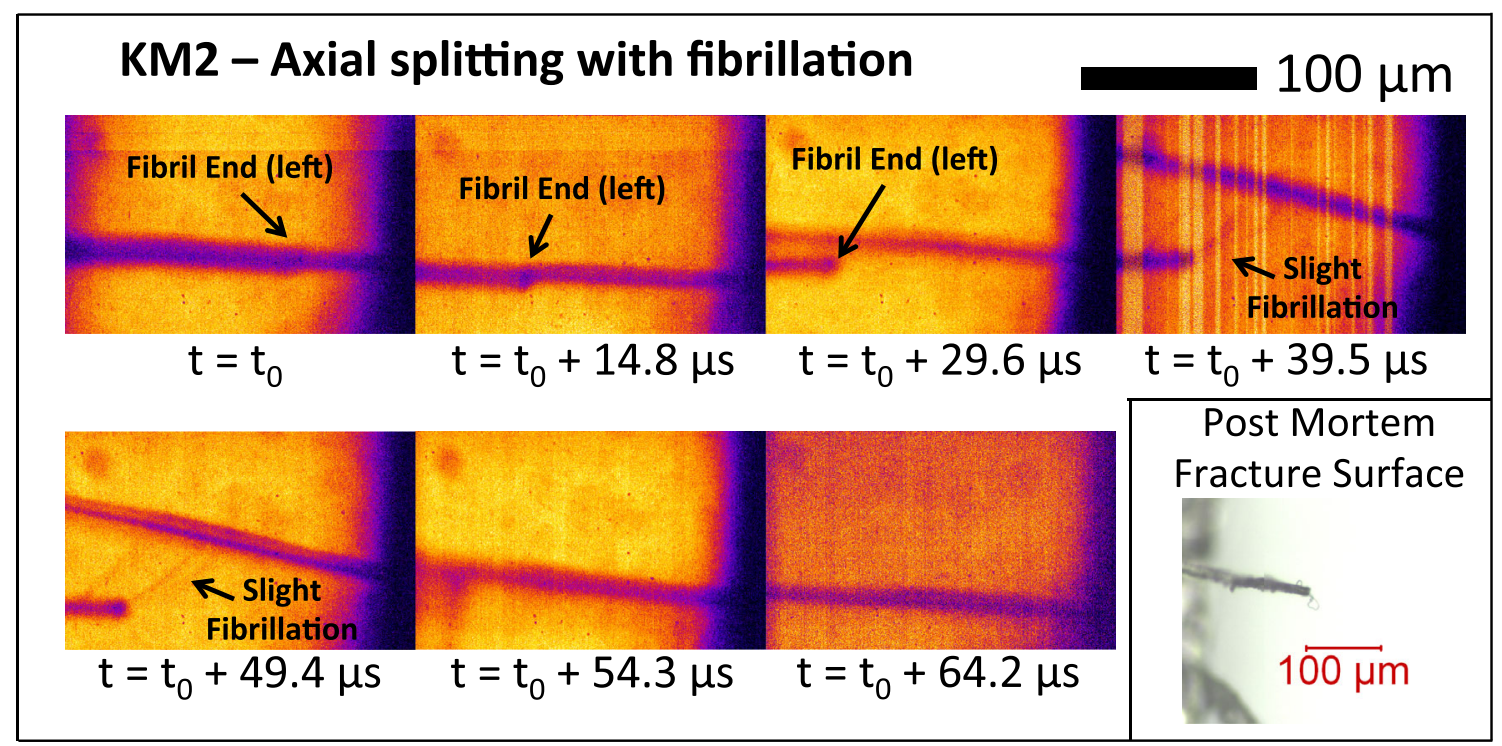

Fig. 6 In situ optical imaging of the Kevlar ${ }^{\circledR}$ KM2 fiber when loaded in high-rate tension. Clear axial splitting phenomenon, which can be deduced from the post-mortem failure surface, is shown in the bottom right inset

majority of the sample gauge length is lost during the duration of the test post initial failure. Therefore, uncovering the correct failure surfaces of said fibers must be done with in situ imaging, as has been shown in the bottom image sets of Figs. 3 and 4. It can be seen from the micrographs presented in Figs. 3 and 4 that the S-2 Glass ${ }^{\circledR}$ fiber does not undergo a total disintegration process upon fracture, but breaks solely in one location. The disintegration or explosive failure phenomenon most likely occurs post initial fiber fracture, thus rendering any further fiber breakage in the now two pieces of disconnected gauge length useless in any meaningful energy transfer process. In order to ascertain a better understanding of the rupture surface within S-2 Glass ${ }^{\circledR}$ (as well as any other fine fiber type), it is apparent that higher levels of both spatial and temporal resolution are needed for a more in depth, proper analysis.

Inherent in PCI is the capability of increasing the level of contrast present in the image via moving the scintillator farther away from the sample, thereby altering the defocusing distance $D$,

$D=\frac{d \times l}{d+l}$

wherein $d$ and $l$ represent the sample-to-detector and sample-to-scintillator distances, respectively [27]. Thus, with changes in $D$, the resolution developed in the sample is then altered, as the radius $r_{F}$ of a region in the sample corresponding to a point in the image is defined by,

$r_{F}=\sqrt{\lambda D}$ wherein $\lambda$ represents the wavelength of the illumination source [27]. Careful attention must be paid to the location of the detection system with respect to the sample so as to achieve an adequate image resolution while still ensuring a reasonable level of contrast. With proper sample-detector positioning, camera quality thereby becomes the limiting factor of spatial resolution in the experimental setup. The spatial resolution of the imaging technique could be increased with a decrease in pixel size or image magnification, albeit there is still a limitation of the scintillator, as it converts X-rays to light, thereby forcing light as the detected information. Other options include using a pixel array detector (PAD) that specifically detects X-rays, thus the spatial resolution limit becomes the pixel size of the PAD, or possibly projection X-ray microscopy, which utilizes Kirkpatrick-Baez optics. Of equal importance is the capability to increase the temporal resolution while imaging the failure process. As previously mentioned, the hybrid mode was used in this study, which limits the possible framing rate to $\sim 270 \mathrm{~K}$ fps. Due to the extremely fine nature of the fibers of interest, relatively few X-rays with low energy are needed to illuminate the sample. Thus, alternate electron fill modes offered at APS could be used to image the failure process, such as the standard 24-bunch mode, which allows for a maximum framing rate of $6.52 \mathrm{M}$ fps or even possibly the 1296-bunch mode, which could allow for a theoretical framing rate of $352.2 \mathrm{M}$ fps [28]. Indeed, like the spatial resolution, the temporal resolution of the imaging process is limited by the camera system rather than the illumination source itself. 


\section{Conclusions}

In situ imaging of the fracture process for three different archetype fiber types has been performed utilizing two different experimental approaches, namely optical light microscopy and PCI. While both techniques have yielded important information regarding the failure phenomenon from each tested material class of fiber, it is apparent that the light microscopy technique has reached a near maximum performance, and further increases in spatial resolution are limited due to the inherent wavelength of the illumination source itself. Thus, the efficacy of utilizing PCI for fiber fracture analysis has been tested, yielding promise for the approach, as large increases in spatial resolution are possible, providing that the proper electron beam run mode, camera, and magnification lenses are utilized. Furthermore, the PCI technique more easily allows for greater temporal resolution, as each passing of an electron bunch provides the X-ray intensity necessary to sufficiently saturate the imaging detector. This is in contrast to using optical illumination, which is currently limited to the photon flux of a single flash source, thereby causing a decrease in detector saturation at increased strain rates. At the current status, basic understanding of the fracture behavior has been realized for all three fiber types. Kevlar $^{\circledR}$ KM2 undergoes fibrillation, which is defined solely by the degree to which it fibrillates. The hypothesis of necking during the failure process of this $\operatorname{Kevlar}^{\circledR}$ fiber type is not seen in any of the in situ tests, rather axial splitting is detected in situ, resulting in a post-mortem fracture surface that can be easily misinterpreted as necking. The Dyneema ${ }^{\circledR}$ SK76 fiber was seen to fibrillate, and on average, the fibrillation was less pronounced than the Kevlar ${ }^{\circledR}$ KM2 fiber. Typical fibrillation was seen to include plate-like formation, most likely evolving from the skincore structure of the fiber. Few of the Dyneema ${ }^{\circledR}$ SK76 tensile tests did exhibit mass fibrillation, but these tests were uncommon, thus should not define the typical failure phenomenon. S-2 Glass ${ }^{\circledR}$ was seen to exhibit brittle fracture at one location along the fiber length, being opposed to the previous post-mortem analysis that described the failure process as full disintegration. Further failure past the initial brittle fracture is thus concluded to occur post rupture due to fiber snap-back or bending. Most importantly, the efficacy of implementing the PCI technique for fiber fracture has been demonstrated, yielding promise in future analysis of fiber rupture with increased levels of both temporal and spatial resolution as opposed to typical highrate light microscopy.

Acknowledgments We appreciate professional help from A. Deriy and B. Glagola (ANL) with certain technical and safety aspects of our experiments at 32ID. Use of the Advanced Photon Source, an Office of Science User Facility operated for the US Department of Energy (DOE) Office of Science by Argonne National Laboratory, was supported by the US DOE under Contract No. DE-AC02-06CH11357. Funding for this work has been provided by the Office of Naval Research, US ARMY PEO Soldier program, and the Materials in Extreme Dynamic Environments (MEDE) Collaborative Research Alliance for testing Dyneema ${ }^{\circledR}$ SK76, Kevlar ${ }^{\circledR}$ KM2, and S-2 Glass ${ }^{\circledR}$ fiber, respectively. The first author would also like to thank the NDSEG fellowship for graduate research funding.

\section{References}

1. Yang H (1989) Aromatic high strength fibers. Wiley, New York

2. Yang HH (1993) Kevlar $\left.^{(}\right)$Aramid Fiber. Wiley, Chichester

3. Prevorsek D (1996) High technology fibers part D, vol 3. Marcel Dekker, Inc, New York, pp 1-170

4. Hearle J (ed) (2001) High-performance fibres. Woodhead Publishing Limited, Cambridge

5. Elices M, Llorca J (eds) (2002) Fiber fracture. Elsevier, Amsterdam

6. Hearle J (2002) Fracture of highly oriented, chain- extended polymer fibres. Fiber fracture. Elsevier Science Ltd, Oxford

7. Gupta PK (1994) Fractography of fiberglass. Fractography of glass. Springer, New York

8. Northolt M (1974) X-ray diffraction study of poly(p-phenylene terephthala-mide) fibres. Eur Polym J 10(9):799-804

9. Deteresa SJ, Allen SR, Farris RJ, Porter RS (1984) Compressive and torsional behaviour of Kevlar ${ }^{\circledR} 49$ fibre. J Mater Sci 19:57-72

10. DeTeresa SJ, Porter RS, Farris RJ (1985) A model for the compres- sive buckling of extended chain polymers. J Mater Sci 20:1645-1659

11. Kumar S, Helminiak T (1990) Compressive strength of high performance fibers. SAMPE 26(2):51-61

12. Warner SB (1987) Critical strain to dead bend. J Mater Sci Lett 6:951-952

13. Brand D (2008) Dyneema ${ }^{\circledR}$ high-strength, Dyneema ${ }^{\circledR}$ highstrength, high-modulus polyethylene fiber. Tech. Rep. CIS YA100

14. Hearle J (1998) Atlas of fibre fracture and damage to textiles, 2nd edn. Woodhead Publishing Limited, Boca Raton

15. Gupta PK (2002) Strength of glass fibers. Fiber fracture. Elsevier, Amsterdam

16. Hudspeth M, Claus B, Dubelman S, Black J, Mondal A, Parab N et al (2013) High speed synchrotron X-ray phase contrast imaging of dynamic material response to split hopkinson bar loading. Rev Sci Instrum 84(2):025102-025107

17. Luo SN, Jensen BJ, Hooks DE, Fezzaa K, Ramos KJ, Yeager JD et al (2012) Gas gun shock experiments with single-pulse X-ray phase contrast imaging and diffraction at the advanced photon source. Review Sci Instrum 83(7):073903

18. Ramos KJ, Jensen BJ, Iverson AJ, Yeager JD, Carlson CA, Montgomery DS, Thompson DG, Fezzaa K, Hooks DE (2014) In situ investigation of the dynamic response of energetic materials using IMPULSE at the Advanced Photon Source. J Phys Conf Ser 500:142028

19. Brown EN, Furmanski J, Ramos KJ, Dattelbaum DM, Jensen BJ, Iverson AJ, Carlson CA, Fezzaa K, Gray GT III, Patterson BM, Trujillo CP, Martinez DT, Pierce TH (2014) High-density polyethylene damage at extreme tensile conditions. J Phys Conf Ser 500:112011

20. Jensen BJ, Ramos KJ, Iverson AJ, Bernier J, Carlson CA, Yeager JD, Fezzaa K, Hooks DE (2014) Dynamic experiment using IMPULSE at the advanced photon source. J Phys Conf Ser 500:042001 
21. Ramos KJ, Jensen BJ, Yeager JD, Bolme CA, Iverson AJ, Carlson CA, Fezzaa K (2014) Investigation of Dynamic Material Cracking with In Situ Synchrotron-Based Measurements Dynamic Behavior of Materials, Volume 1. Conference Proceedings of the Society for Experimental Mechanics Series 2014, 413-420

22. Astm C 1557-03 (2008) Standard test method for tensile strength and Young's modulus of fibers. Conshohocken, ASTM International

23. Riekel C, Dieing T, Engström P, Vincze L, Martin C, Mahendrasingam A (1999) X-ray microdiffraction study of chain orientation in poly(p-phenylene terephthalamide). Macromolecules 32(23):7859-7865

24. Graham JF, McCague C, Warren OL, Norton PR (2000) Spatially resolved nanomechanical properties of Kevlar ${ }^{\circledR}$ fibers. Polymer 41(12):4761-4764
25. Roth S, Burghammer M, Janotta A, Riekel C (2003) Rotational disorder in poly(p-phenylene terephthalamide) fibers by $\mathrm{x}$-ray diffraction with a $100 \mathrm{~nm}$ beam. Macromolecules 36(5):1585-1593

26. Davies RJ, Burghammer M, Riekel C (2006) Simultaneous microfocus raman and microfocus xrd: probing the deformation of a single high-performance fiber. Macromolecules 39(14):4834-4840

27. Cloetens P, Ludwig W, Baruchel J, Guigay JP, Pernot-Rejmánková J, Salomé-Pateyron M, Schlenker M, Buffière J, Maire E, Peix G (1999) Hard x-ray phase imaging using simple propagation of a coherent synchrotron radiation beam. J Phys D Appl Phys 32:A145

28. Storage ring operation modes (2015). http://www.aps.anl.gov 